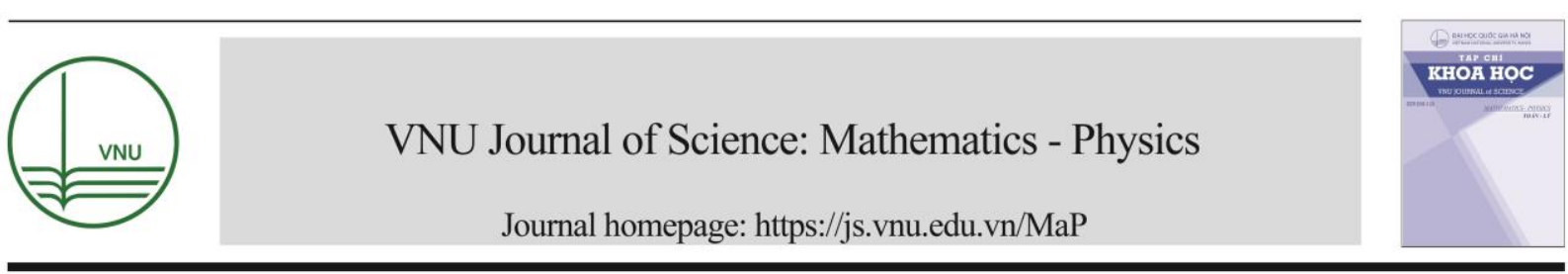

\title{
Molecular-field Study on Sn Substituted Yttrium Iron Garnet
}

\author{
Nguyen Phuc Duong* \\ ITIMS, Hanoi University of Science and Technology, \\ No. 1, Dai Co Viet, Hai Ba Trung, Hanoi, Viet Nam \\ Received 31 January 2018 \\ Revised 28 February 2018; Accepted 28 February 2018
}

\begin{abstract}
Samples with the compositions $\mathrm{Y}_{2.9} \mathrm{Ca}_{0.1} \mathrm{Fe}_{4.9} \mathrm{Sn}_{0.1} \mathrm{O}_{12}$ and $\mathrm{Y}_{3} \mathrm{Fe}_{4.9} \mathrm{Sn}_{0.1} \mathrm{O}_{12}$ were prepared by using a sol-gel technique. The influence of the substituted non-magnetic cations with different valences in the structural and magnetic properties was studied. X-ray diffraction and fieldemission electron scanning microscope techniques were used to study the crystal structure and morphology. Magnetization curves in fields up to $10 \mathrm{kOe}$ and in temperature range from $80 \mathrm{~K}$ to $570 \mathrm{~K}$ were measured by means of a vibrating sample magnetometer. Saturation magnetization as a function of temperature of the two samples was analyzed based on the molecular-field theory from that models for site occupancy and valence states of cations in the crystal structures were derived.
\end{abstract}

Keywords: Yttrium iron garnet; $\mathrm{Ca}$ and $\mathrm{Sn}$ substitution; magnetization; Curie temperature; molecular-field calculation

\section{Introduction}

Yttrium iron garnet (YIG) crystallizes in cubic structure with the space group Ia3d. $\mathrm{Y}^{3+}$ ions occupy the dodecahedral sites $24 c$ while $\mathrm{Fe}^{3+}$ ions are distributed over the octahedral sites $16 a$ and tetrahedral sites $24 d$ forming two magnetic sublattices [a] and (d), respectively. Stoichiometric YIG is a good insulator with room temperature resistivity greater than $10^{12} \Omega \mathrm{cm}[1]$ and has been widely used in high frequency applications [2]. By doping or substituting cations which have valences other than 3, charge carriers may arise in the materials and n- or p-type conductivity can be obtained. Donor or acceptor centers are created by substituting cations with valence greater (e.g., $\mathrm{Si}^{4+}, \mathrm{Ge}^{4+}, \mathrm{Sn}^{4+}, \mathrm{Zr}^{4+}$, $\mathrm{Sb}^{5+}, \mathrm{Mo}^{6+}$ etc. $)$ and less $\left(\mathrm{Ca}^{2+}, \mathrm{Pb}^{2+}, \mathrm{Zn}^{2+}, \mathrm{Mg}^{2+}\right.$ etc. $)$ than 3 , respectively. Conductive iron garnets are promising for other applications such as optical sensors and gas sensors [3-5].

Substitution by other elements also leads to a change in magnetic properties. In the pure YIG compound, the $\mathrm{Fe}^{3+}$ ions in the $a$ and $d$ sublattices order in the ferrimagnetic structure with the ideal

\footnotetext{
*Tel.: 84-915527063.

Email: duong@itims.edu.vn

https//doi.org/ 10.25073/2588-1124/vnumap.4260
} 
net moment value at the ground state of $5 \mu_{\mathrm{B}}$ per formula unit according to the Néel configuration. The Curie temperature $\left(T_{\mathrm{C}}=565 \mathrm{~K}\right)$ is governed by the strongest intersublattice $\mathrm{Fe}^{3+}{ }_{\mathrm{a}}-\mathrm{Fe}^{3+}{ }_{\mathrm{d}}$ interaction [6]. Previous studies showed that by using molecular-field theory applied for the Néel configuration, the evolution of the magnetic sublattices upon substituting non-magnetic ions can be anticipated [7].

In this paper we investigate the effect of $\mathrm{Sn}$ substitution on the magnetization and Curie temperature of YIG samples prepared by sol-gel route in the light of molecular-field theory. Two compositions $\mathrm{Y}_{2.9} \mathrm{Ca}_{0.1} \mathrm{Fe}_{4.9} \mathrm{Sn}_{0.1} \mathrm{O}_{12}$ and $\mathrm{Y}_{3} \mathrm{Fe}_{4.9} \mathrm{Sn}_{0.1} \mathrm{O}_{12}$ are considered. In the first composition, the same molar amounts of $\mathrm{Ca}^{2+}$ and $\mathrm{Sn}^{4+}$ are substituted for $\mathrm{Y}^{3+}$ and $\mathrm{Fe}^{3+}$, respectively, therefore the total positive and negative charges in a formula unit are balanced out. In the second composition, only $\mathrm{Sn}^{4+}$ is substituted for $\mathrm{Fe}^{3+}$ hence in order to satisfy the charge neutrality condition the same amount of iron ions will have valence 2+. By comparing experimental and calculation magnetization data, information on the chemical properties of the materials such as homogeneity, stoichiometry, cation distribution and valence state of iron cations can be deduced. These chemical factors are important information for tailoring desired electrical properties the above mentioned applications.

\section{Experimental}

Samples with compositions $\mathrm{Y}_{2.9} \mathrm{Ca}_{0.1} \mathrm{Fe}_{4.9} \mathrm{Sn}_{0.1} \mathrm{O}_{12}$ and $\mathrm{Y}_{3} \mathrm{Fe}_{4.9} \mathrm{Sn}_{0.1} \mathrm{O}_{12}$ were prepared by using citrate sol-gel technique and are denoted as YIG-Ca-Sn and YIG-Sn, respectively. Details of the sample preparation were described elsewhere [8]. Amounts of $\mathrm{Y}\left(\mathrm{NO}_{3}\right)_{3}, \mathrm{Fe}\left(\mathrm{NO}_{3}\right)_{3}, \mathrm{Ca}\left(\mathrm{NO}_{3}\right)_{2}$ and $\mathrm{Sn}\left(\mathrm{NO}_{3}\right)_{4}$ with desired molar ratios were dissolved completely in deionized water. The obtained aqueous solution was poured into citric acid with the total cations/citric acid molar ratio is 1:1. Ammonium hydroxide in aqueous form was added to the mixed solutions and the $\mathrm{pH}$ of the solutions was adjusted to about 10 . The mixtures were stirred at $600 \mathrm{rpm}$ and slowly evaporated at $110^{\circ} \mathrm{C}$ to form gels. The gels were dried at $330^{\circ} \mathrm{C}$ for more than $2 \mathrm{~h}$ in order to form xerogels. The samples were obtained after annealing the products at $900^{\circ} \mathrm{C}$ in $8 \mathrm{~h}$.

$\mathrm{X}$-ray diffraction $(\mathrm{Cu}-\mathrm{K} \alpha$, Siemens D-5000) was employed to identify the crystal Structure of the samples at room temperature. Field Emission-Scanning Electron Microscopy (FESEM) (JEOL JSM$7600 \mathrm{~F}$ ) was used to examine the grain size and morphology. Magnetization curves were measured using a vibrating sample magnetometer (VSM, DMS 880) in the temperature range of $80-570 \mathrm{~K}$ and in applied magnetic fields up to $10 \mathrm{kOe}$. For the magnetic measurements, the powders were pressed in the forms of platelets.

\section{Results and disscution}

\subsection{Crystal structure and morphological analysis}

The XRD patterns of the samples shown Fig. 1 indicate that the samples are single phase of which all the observed diffraction peaks can be well indexed within the standard garnet structure. For comparison, the pure YIG sample was also prepared in the same fabrication conditions and its XRD pattern was recorded. The diffraction peaks of the substituted samples are found to shift to lower Bragg angles. The unit cell parameter $a$ was computed using the interplanar distance $d$ and the respective $(h, k, l)$ parameters. The lattice constant values are listed in Table 1 . The results show that the lattice constant of the substituted samples increases. This phenomenon can be explained due to the larger radii of $\mathrm{Ca}^{2+}$ and $\mathrm{Sn}^{4+}$ ions compared to $\mathrm{Y}^{3+}$ and $\mathrm{Fe}^{3+}$, respectively. For examples, in octahedral 
sites, $r_{\mathrm{Sn} 4+}=0.69 \AA, r_{\mathrm{Fe} 3+}=0.63 \AA$ and in dodecahedral sites, $r_{\mathrm{Ca} 2+}=1.48 \AA, r_{\mathrm{Y} 3+}=1.015 \AA$ [9]. The crystallite size of the samples was calculated based on the width of the (420) peak using the DebyeScherrer formula. An average crystallite size DXRD of $\sim 35 \mathrm{~nm}$ is deduced for all the samples. The microstructure of the samples was characterized by FESEM. The results show that the grain sizes of both samples are in order of few hundred nanometers and the materials are melted and the grains are joined in networks with spongy structures.

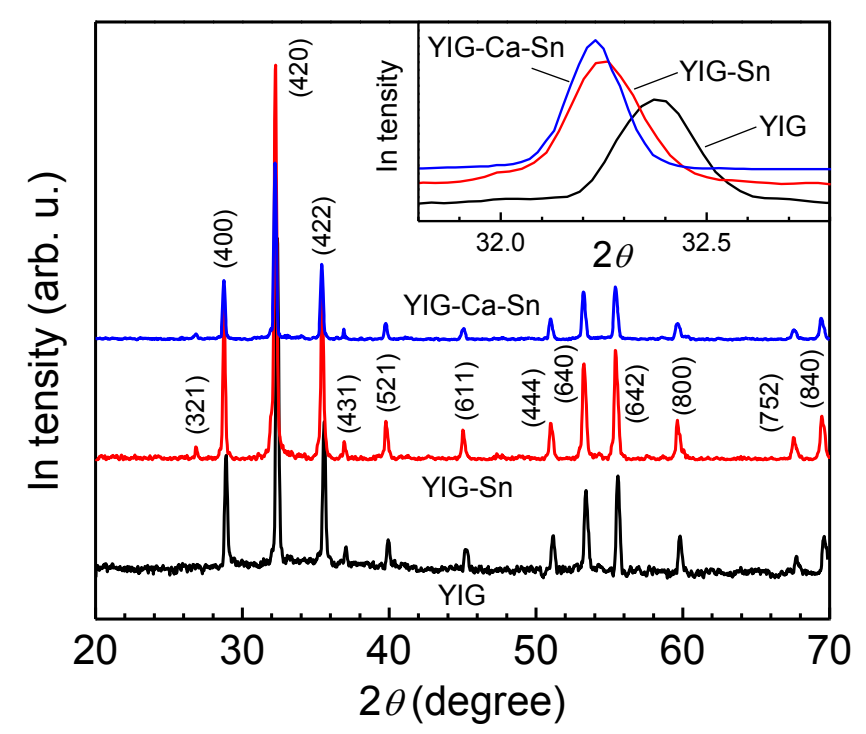

Fig. 1. XRD paterrns of YIG, YIG-Ca-Sn and YIG-Sn samples. The inset shows the (420) peak of the samples in magnified scale.

Table 1. Lattice parameter a, Curie temperature $T_{\mathrm{C}}$ and extrapolated magnetic moment at zero kelvin $m(0)^{\mathrm{ext}}$ of the samples.

\begin{tabular}{llll}
\hline & $a(\AA)$ & $T_{C}(K)$ & $\begin{array}{l}m(0)^{e x t} \\
\left(\mu_{B} / f \cdot u .\right)\end{array}$ \\
\hline YIG & 12.383 & & \\
YIG-Sn & 12.408 & 538 & 4.58 \\
YIG-Ca-Sn & 12.424 & 547 & 4.3 \\
\hline
\end{tabular}

\subsection{Magnetic characterization}

The magnetization curves $M-H$ were measured by means of VSM. For demonstration, the magnetization loops at several temperatures are presented in Fig. 2 for YIG-Ca-Sn and YIG-Sn. A common feature of the curves at different temperatures is that the magnetization approaches to saturation above $\sim 2.5 \mathrm{kOe}$ and with further increasing magnetic field the saturation state is attained with negligible magnetic susceptibility. The 'technical' saturation magnetization $M_{\mathrm{s}}$ values were determined based on the flat part of the curves. The temperature dependence of the saturation magnetization expressed in emu per gram (emu/g) is shown in Fig. 3. From these curves, the Curie temperatures $T_{\mathrm{C}}$ were determined as the temperature at which $M_{\mathrm{s}}$ vanishes. 

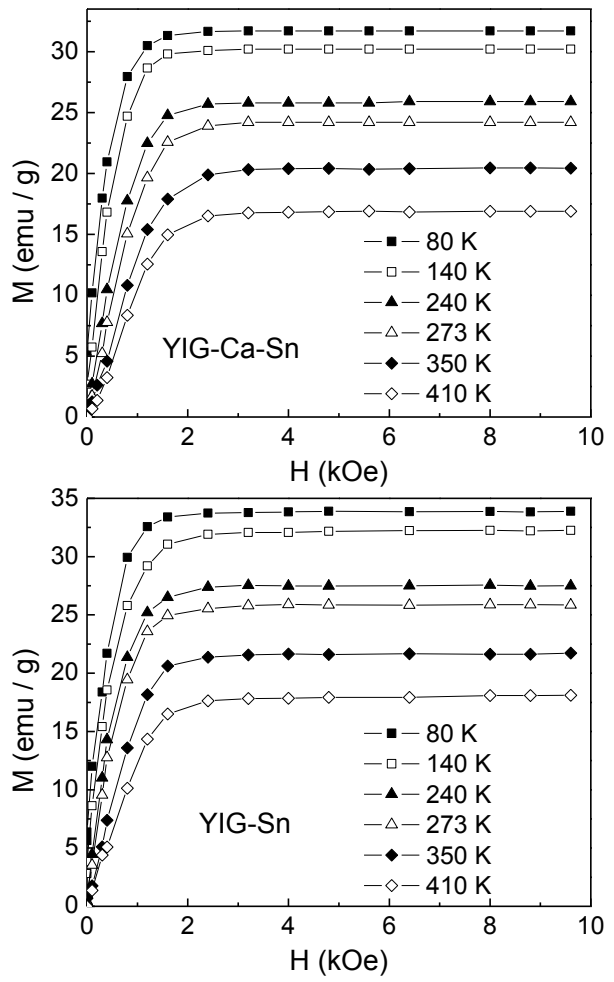

Fig. 2. Magnetization curves of YIG-Ca-Sn (up) and YIG-Sn (down) samples at several temepratures.

Table 2. Molecular-field parameters and calculated Curie temperature $T_{\mathrm{C}}{ }^{\mathrm{cal}}$ and magnetic moment at zero kelvin $m(0)^{\mathrm{cal}}$ for the $\mathrm{Y}_{3}\left[\mathrm{Fe}_{2-x} R_{x}\right]\left(\mathrm{Fe}_{3}\right)$ and $\mathrm{Y}_{3}\left[\mathrm{Fe}_{2}\right]\left(\mathrm{Fe}_{3-y} Q_{y}\right)$ model compounds where $R$ and $Q$ are non-magnetic cations.

\begin{tabular}{|c|c|c|c|c|c|}
\hline$x$ & 0 & 0.05 & 0.1 & 0.15 & 0.2 \\
\hline $\begin{array}{l}N_{\mathrm{ad}} \\
\left(\mathrm{mol} . \mathrm{cm}^{-3}\right)\end{array}$ & 97 & 96.4 & 95.8 & 95.2 & 94.6 \\
\hline $\begin{array}{l}N_{\mathrm{aa}} \\
\left(\mathrm{mol} . \mathrm{cm}^{-3}\right)\end{array}$ & -65 & -65 & -65 & -65 & -65 \\
\hline $\begin{array}{l}N_{\mathrm{dd}} \\
\left(\mathrm{mol} . \mathrm{cm}^{-3}\right)\end{array}$ & -30.4 & -29.8 & -29.1 & -28.5 & -27.8 \\
\hline$T_{\mathrm{C}}^{\mathrm{cal}}(\mathrm{K})$ & 556 & 552 & 549 & 542 & 538 \\
\hline $\begin{array}{l}m(0)^{\mathrm{ext}} \\
\left(\mu_{\mathrm{B}} / \text { f.u. }\right)\end{array}$ & 5.0 & 5.25 & 5.5 & 5.75 & 6.0 \\
\hline$y$ & 0 & 0.05 & 0.1 & 0.15 & 0.2 \\
\hline $\begin{array}{l}N_{\mathrm{ad}} \\
\left(\mathrm{mol} . \mathrm{cm}^{-3}\right)\end{array}$ & 97 & 96.4 & 95.7 & 95.1 & 94.5 \\
\hline $\begin{array}{l}N_{\mathrm{aa}} \\
\left(\mathrm{mol} . \mathrm{cm}^{-3}\right)\end{array}$ & -65 & -63.6 & -62.3 & -60.9 & -59.5 \\
\hline $\begin{array}{l}N_{\mathrm{ad}} \\
\left(\mathrm{mol} \mathrm{cm}^{-3}\right)\end{array}$ & -30.4 & -30.4 & -30.4 & -30.4 & -30.4 \\
\hline$T_{\mathrm{C}}^{\mathrm{c} a}(\mathrm{~K})$ & 556 & 551 & 549 & 545 & 542 \\
\hline $\begin{array}{l}m(0)^{\mathrm{ext}} \\
\left(\mu_{\mathrm{B}} / \text { f.u. }\right)\end{array}$ & 5.0 & 4.75 & 4.5 & 5.24 & 4.0 \\
\hline
\end{tabular}



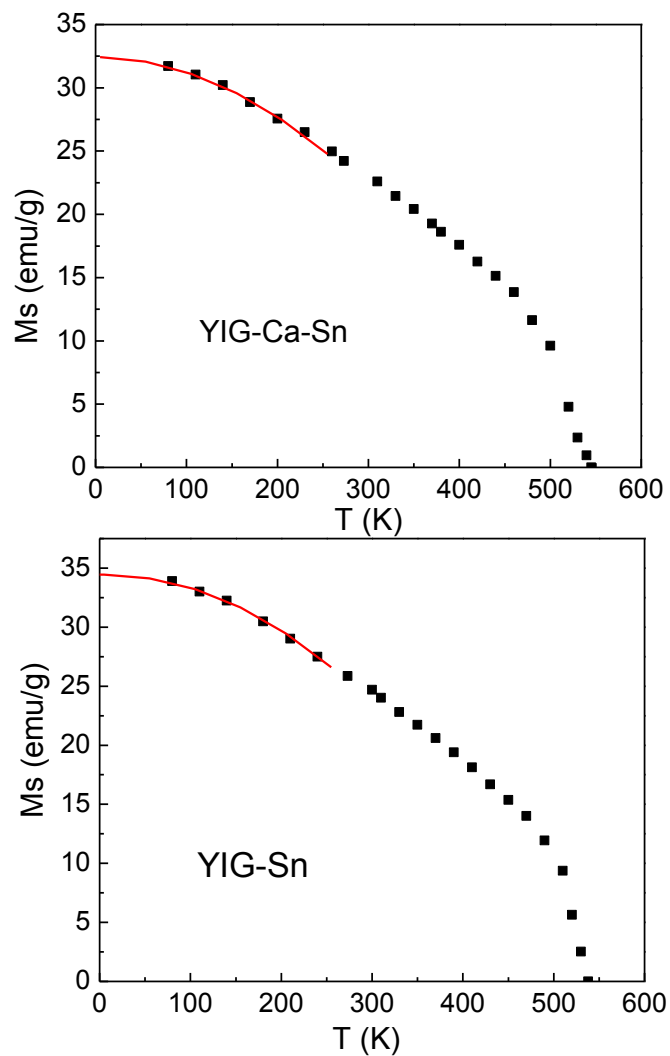

Fig. 3. Temperature dependence of saturation magentization $M_{\mathrm{s}}$ of YIG-Ca-Sn (up) and YIG-Sn (down) samples.

The lines are fitting curves according to eq. (2).

The calculation of the $M_{\mathrm{s}} v s T$ curve of YIG has been achieved first by Anderson [10] and later by Dionne [7] on the basis of the two-sublattice model of Néel [11]. The intra- and intersubattice interactions between magnetic moments in [a] and (d) sublattices are characterized by the exchange coefficients $N_{\text {aa }}, N_{\text {dd }}$ and $N_{\text {ad }}$ in molecular-field approximation (MFA). Via the MFA fit which best satisfies all sets of experimental data reported in [7,12-14], Dionne found that $N_{\mathrm{ad}}=97.0 \mathrm{~mol} / \mathrm{cm}^{3}, N_{\mathrm{aa}}$ $=-65.0 \mathrm{~mol} / \mathrm{cm}^{3}$, and $N_{\mathrm{dd}}=-30.4 \mathrm{~mol} / \mathrm{cm}^{3}$. By further examining the magnetization curves of various compositions with general formula $\mathrm{Y}_{3}\left[\mathrm{Fe}_{2-x} R_{x}\right]\left(\mathrm{Fe}_{3-y} Q_{y}\right)$ in which $\mathrm{Fe}$ is substituted by non-magnetic elements $\left(R\right.$ and $Q=\mathrm{Sc}^{4+}, \mathrm{Sn}^{4+}, \mathrm{Zr}^{4+}, \mathrm{In}^{3+}, \mathrm{Al}^{3+}, \mathrm{Ga}^{3+}$, etc.), Dionne found that the exchange parameters decrease with increasing magnetic dilution levels and the rate depends on whether magnetic dilution takes place at $a$ or $d$ sublattice. Generalized formulas for the changes of the exchange coefficients as functions of non-magnetic substitutions were established as follows [7]

$$
\begin{aligned}
& N_{\mathrm{ad}}=97.0(1-0.125 x-0.127 y) \\
& N_{\mathrm{aa}}=-65.0(1-0.42 y) \\
& N_{\mathrm{dd}}=-30.4(1-0.43 x) \\
& \text { with } x \leq 0.70 \text { and } y \leq 1.95 .
\end{aligned}
$$



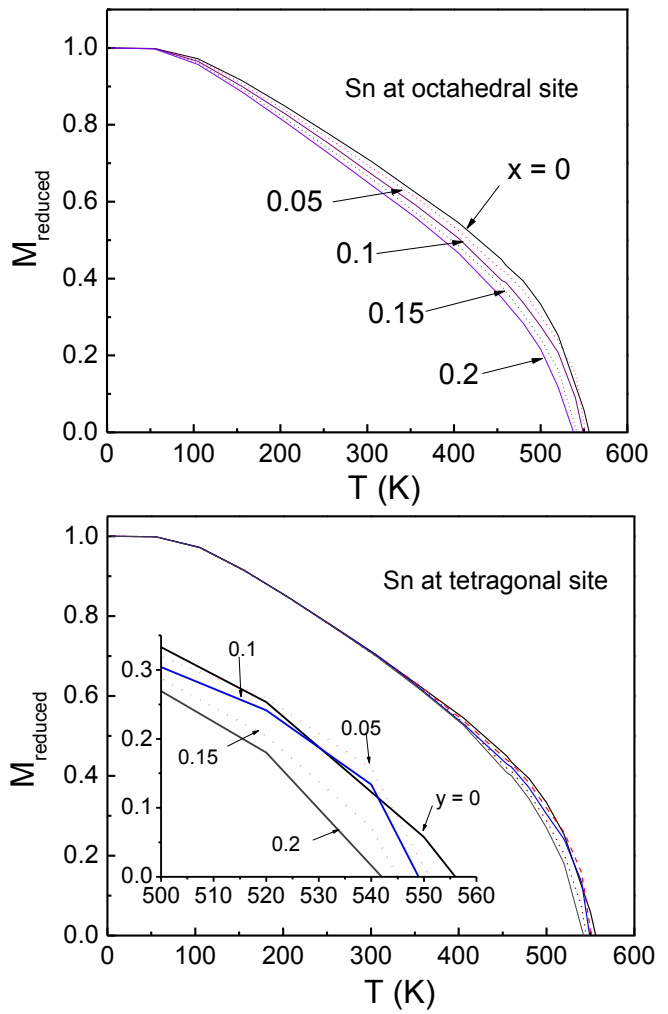

Fig. 4. Molecular-field calcualtions for $M_{\text {reduced }} v s T$ of $\mathrm{Y}_{3}\left[\mathrm{Fe}_{2-x} R_{x}\right]\left(\mathrm{Fe}_{3}\right)$ (up) and $\mathrm{Y}_{3}\left[\mathrm{Fe}_{2}\right]\left(\mathrm{Fe}_{3-y} Q_{y}\right)$ (down) with $x$, $y=0,0.05,0.15$ and 0.2 . The inset shows the magnified parts of the curves near Curie temperature.

Using the fitting parameters derived from eqs. (1) for two cases of cation distribution $\mathrm{Y}_{3}\left[\mathrm{Fe}_{2-}\right.$ $\left.{ }_{x} R_{x}\right]\left(\mathrm{Fe}_{3}\right)$ and $\mathrm{Y}_{3}\left[\mathrm{Fe}_{2}\right]\left(\mathrm{Fe}_{3-y} Q_{y}\right)$, we generated the $m v s T$ curves for $x$ and $y$ taking the values of 0.05 , $0.1,0.15$ and 0.2 . Here $m$ is the net magnetic moment expressed in $\mu_{\mathrm{B}} /$ f.u. The fitting was implemented following the procedure described by Dionne in [7]. Fig. 4 reports the calculation results for the temperature dependence of reduced magnetic moment $\left(m_{\text {reduced }}=m(T) / m(0)\right)$ for the two cases. The fitting parameters and calculated $m(0)$ and $T_{\mathrm{C}}$ values are listed in Table 2 . It is seen that for $y$ substitution the magnetic moment decreases faster with increasing temperature while for $x$ substitution, the $m_{\text {reduced }} v s T$ curves are almost coincide in a wide temperature range. Following the Néel spin configuration, the net moment at $0 \mathrm{~K}$ is determined as $m(0)=m_{\mathrm{d}}-m_{\mathrm{a}}$, where $m_{\mathrm{a}}=(2-$ $x) g_{\mathrm{Fe}} S^{\mathrm{Fe} 3+}$ and $m_{\mathrm{d}}=(3-y) g_{\mathrm{Fe}} S^{\mathrm{Fe} 3+}\left(g_{\mathrm{Fe}}=2\right.$ and $\left.S^{\mathrm{Fe} 3+}=5 / 2\right)$. Compared to YIG, $m(0)$ of the substituted compounds increases with $x$ and decreases with $y$ (see Table 2).

The part of the $m_{\text {reduced }} v s T$ curves in low temperature region can also be well fitted by using a Bloch-type equation:

$$
m(T) / m(0)=1-B T^{\alpha}
$$

with the exponent values $\alpha \approx 2$.

For comparison, the saturation magnetization at $0 \mathrm{~K}, M_{\mathrm{s}}(0)^{\mathrm{ext}}$, of the investigated samples were also determined via fitting the low-temperature part of magnetization curves in the range 80-250 K using eq. (2) with $\alpha=2$. The fitting curves are shown in Fig. 3. The $M_{\mathrm{s}}(0)^{\text {ext }}$ decreases with $\mathrm{Sn}$ substitution which reveals that in both samples $\mathrm{Sn}^{4+}$ ions occupy the $d$ sites. The extrapolated magnetic moments in the ground state were calculated according to the formula: $m(0)^{\mathrm{ext}}=M_{\mathrm{s}}(0)^{\mathrm{ext}} \times W / 5585$, 
where $W$ is molar mass and the results are listed in Table 1 . For the sample YIG-Ca-Sn, the $m_{\text {reduced }} v s$ $T$ curve follows very well the behavior of the curves obtained via molecular-field calculations for the compositions $y=0.1$ and 0.15 in wide temperature range as seen in the upper panel of Fig. 5. The $m(0)^{\text {ext }}$ and $T_{\mathrm{C}}$ values of this sample are slightly larger and smaller than those calculated for $y=0.15$ and 0.1 , respectively. This observation suggests a deviation in Sn concentration of the sample YIG$\mathrm{Ca}-\mathrm{Sn}$ compared to the nominal composition. On the other hand, the magnetic behavior is quite distinct for the sample YIG-Sn. The $m(0)^{\text {ext }}$ value of this sample is considerably larger than the $m(0)$ value calculated for the same magnetic dilution level $(y=0.1)$ while the $T_{\mathrm{C}}$ value is strongly reduced (see Tables 1 and 2). In the case of YIG-Ca-Sn, co-substituting $\mathrm{Ca}^{2+}$ and $\mathrm{Sn}^{4+}$ for $\mathrm{Y}^{3+}$ and $\mathrm{Fe}^{3+}$, respectively, with the same molar amounts leads to a balance between the positive charge of the metal ions and the negative charge of the oxygen anions. Hence, the Fe ions in the sample would have the same valence 3+ as in YIG compound. However, in the case of YIG-Sn, only $\mathrm{Sn}^{4+}$ is substituted for $\mathrm{Fe}^{3+}$, therefore, in order to attain charge neutrality some of the Fe ions should be $2+\left(S^{\mathrm{Fe} 2+}=2\right)$. In this condition the molar fraction of $\mathrm{Fe}^{2+}$ is also 0.1. In addition, if the amount of $\mathrm{Fe}^{+2}$ locates at the $a$ sites, the total magnetic moment will increases as observed experimentally. According to the Néel model, the magnetic moment of the composition can be estimated as $m(0)=(3-y) g_{\mathrm{Fe}} S^{\mathrm{Fe} 3+}-(2-y) g_{\mathrm{Fe}} S^{\mathrm{Fe} 3+}-$ $y g_{\mathrm{Fe}} S^{\mathrm{Fe} 2+}$ which equals to $4.6 \mu_{\mathrm{B}} /$ f.u. for $y=0.1$. This value is in good agreement with the extrapolated value deduced for the sample YIG-Sn (Table 1). Also, due to Fe ions in the $a$ sublattice is partially $\mathrm{Fe}^{2+}$ which has lower spin number, the intra- and intersublattice interactions in the compound will decreases, leading to a decrease of the Curie temperature [14]. The lower panel of Fig. 4 shows the MF fit to the $m_{\text {reduced }} v s T$ curve for the sample YIG-Sn. Best fitting curve is obtained with the set of the parameters $N_{\mathrm{ad}}=92 \mathrm{~mol} . \mathrm{cm}^{3} ; N_{\mathrm{aa}}=-60 \mathrm{~mol} . \mathrm{cm}^{3}$ and $N_{\mathrm{dd}}=-27 \mathrm{~mol} . \mathrm{cm}^{-3}$. These parameters are all smaller than those listed in Table 2 for the case $y=0.1$.
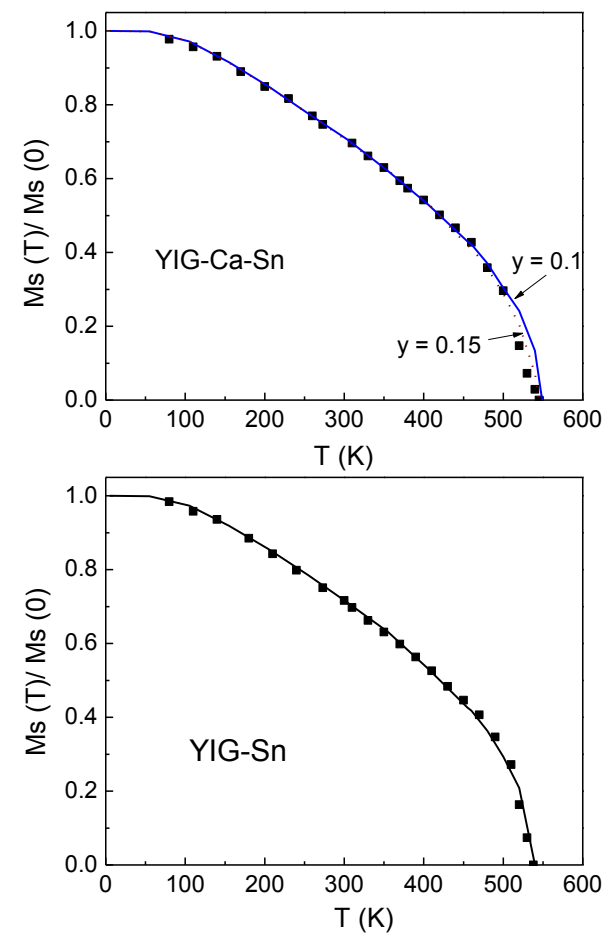

Fig. 5. Temperature dependence of $M_{\mathrm{s}}(T) / M_{\mathrm{s}}(0)$ of YIG-Ca-Sn (up) and YIG-Sn (down) samples. The lines are fitting curves using molecular-field theory. 


\section{Conclusion}

Single-phase YIG-Ca-Sn and YIG-Sn samples were prepared by using sol-gel technique. Crystal structure and magnetization of the samples were studied experimentally. The temperature dependence of the saturation magnetization of the samples was well described using molecular-field theory. Models for the distribution and valence states of the cations in these materials were derived from this study. In the molecular-field calculations, Sn atoms were assumed to reside in the $d$ sites in both samples. In order to explain the high value of magnetization and low Curie temperature of the for YIG-Sn sample, an amount of $\mathrm{Fe}^{2+}$ equal to that of $\mathrm{Sn}^{4+}$ was evoked in the $a$ sublattice.

\section{Acknowledgments}

This work was financially supported by the Vietnam National Foundation for Science and Technology Development under Grant No. 103.02-2016.05.

\section{References}

[1] A. Tucciarone, P. de Gasperis, Electrical properties of iron garnet films, Thin Solid Films 114 (1984) 109-134.

[2] G. Winkler, Magnetic Garnets, Friedr. Vieweg and Sohn, Braunschweig/Wiesbaden, 1981.

[3] A. D'Amico, A. Grilli, A. Paoletti, P. Paroli, A. Tucciarone, Doped yttrium iron garnet for thermistor-bolometers, Mat. Res. Bul. 19 (1984) 347-354.

[4] D. Matatagui, O.V. Kolokoltsev, N. Qureshi, E.V. Mejía-Uriarte, C.L. Ordoñez-Romero, A. Vázquez-Olmos, J.M. Saniger, Magnonic sensor array based on magnetic nanoparticles to detect, discriminate and classify toxic gases, Sens. and Actuators B 240 (2017) 497-502.

[5] V.N. Berzhansky, A.V. Karavainikov, T.V. Mikhailova, A.R. Prokopov, A.N. Shaposhnikov, A.G. Shumilov, N.V. Lugovskoy, E.Yu. Semuk, M.F. Kharchenko, I.M. Lukienko, Yu.M. Kharchenko, V.I. Belotelov, Nano- and micro-scale Bi-substituted iron garnet films for photonics and magneto-optic eddy current defectoscopy, J. Magn. Magn. Mater. 440 (2017) 175-178.

[6] M.A. Gilleo, Ferromagnetic Materials: A Handbook of the Properties of Magnetically Ordered Substances, E.P. Wohlfarth, (Ed.), North-Holland, Amsterdam, vol. 2, 1980 (Chapter 1).

[7] Gerald F. Dionne, Molecular field coefficients of substituted yttrium iron garnets, J. Appl. Phys. 41 (1970) 48744881.

[8] Dao Thi Thuy Nguyet, Nguyen Phuc Duong, Takuya Satoh, Luong Ngoc Anh, Than Duc Hien, Temperaturedependent magnetic properties of yttrium iron garnet nanoparticles prepared by citrate sol-gel, J. Alloys Compd. 541 (2012) 18-22.

[9] R. D. Shannon, Revised Effective Ionic Radii and Systematic Studies of Interatomie Distances in Halides and Chaleogenides, Acta Cryst. A32 (1976) 751-767.

[10] E.E. Anderson, Molecular Field Model and the Magnetization of YIG, Phys. Rev. 134 (1964) A1581-A1585.

[11] L. Néel, Propriétés magnétiques des ferrites ; ferrimagnétisme et antiferromagnétisme, Ann. Phys. 3 (1948) 137198.

[12] E.E. Anderson, The magnetizations of yttrium- and gadolinium-iron garnets, Ph.D. thesis (Physics Department, University of Maryland), 1964.

[13] M.A. Gilleo, Superexchange interaction energy for $\mathrm{Fe}^{3+}-\mathrm{O}^{2-}-\mathrm{Fe}^{3+}$ linkages, Phys. Rev. 109 (1958) 777-781.

[14] S. Krupicka, P. Novak, Ferromagnetic Materials, E.P. Wohlfarth, (Ed.), North-Holland, Amsterdam, vol. 3 , 1982 (Chapter 4). 